\title{
A Note on Gravitational Baryogenesis
}

\author{
H. Mohseni Sadjadi* \\ Department of physics, University of Tehran , \\ P.O.B. 14395-547, Tehran 14399-55961, Iran
}

October 29, 2018

\begin{abstract}
The coupling between Ricci scalar curvature and the baryon number current dynamically breaks CPT in an expanding universe and leads to baryon asymmetry. We study the effect of time dependence of equation of state parameter of the FRW universe on this asymmetry.
\end{abstract}

\section{Introduction}

The origin of the difference between the number density of baryons and anti-baryons is still an open problem in particle physics and cosmology. The measurements of cosmic microwave background [1], the absence of $\gamma$ ray emission from matter- antimatter annihilation [2] and the theory of Big Bang nucleosynthesis [3] indicate that there is more matter than antimatter in the universe. Observational results yield that the ratio of the baryon number to entropy density is approximately $n_{b} / s \sim 10^{-10}$. In [4], it was pointed out that a baryon-generating interaction must satisfy three conditions in order to produce baryons and antibaryons at different rates: (1) baryon number violation; (2) $\mathrm{C}$ and $\mathrm{CP}$ violation; (3) departure from thermal equilibrium.

In [5], by introducing a scalar field coupled to baryon number current it was suggested that the baryon asymmetry may be generated in thermal equilibrium while the CPT invariance is dynamically violated. Similarly, in [6], by introducing an interaction between Ricci scalar curvature and baryon number current which dynamically violates CPT symmetry in expanding Friedman Robertson Walker (FRW) universe, a mechanism for baryon asymmetry was proposed. The proposed interaction shifts the energy of a baryon relative to that of an antibaryon, giving rise to a non-zero baryon number density in thermal equilibrium. The model suggested in [5, 6] was the subject of several studies on gravitational baryogenesis and leptogenesis in different models of cosmology [7].

*mohseni@phymail.ut.ac.ir; mohsenisad@ut.ac.ir 
But, in [6], the problem was restricted to the cases that the equation of state parameter of the universe, $\omega$, is a constant, and the role of time dependence of $\omega$ in baryogenesis was neglected. As a consequence, in this scenario, the baryon number asymmetry cannot be directly generated in radiation dominated epoch. But in [8], in the framework of modified theories of gravity, following the method of [6], it was shown that the baryon asymmetry may be generated even in the radiation dominated era.

In this paper, like [6], we assume that the universe is filled with perfect fluids such as the scalar inflaton field and radiation. The time dependence of $\omega$ is due to the fact that: (1) these components have different equation of state parameters, (2) they interact with each other and, (3) they may have time dependent equation of state parameters. We will study the effect of each of these subjects on time derivative of the Ricci scalar and consequently on baryogenesis. We will elucidate our discussion through some examples.

Natural units $\hbar=c=k_{B}=1$ are used throughout the paper .

\section{The rôle of $\dot{\omega}$ in gravitational baryogenesis}

The mechanism proposed in [6] to generate baryon asymmetry is based on the introduction of a $\mathrm{CP}$ violating interaction between the derivatives of the Ricci scalar and the baryon number current, $J^{\mu}$, given by

$$
\frac{\varepsilon}{M_{\star}^{2}} \int d^{4} x \sqrt{-g}\left(\partial_{\mu} R\right) J^{\mu},
$$

where $M_{\star}$ is a cutoff characterizing the scale of the energy in the effective theory and $\varepsilon= \pm 1$. To obtain the chemical potential for baryon $\left(\mu_{B}\right)$ and antibaryons $\left(\mu_{\bar{B}}\right)$ for spatially constant $R$, we use

$$
\frac{1}{M_{\star}^{2}}\left(\partial_{\mu} R\right) J^{\mu}=\frac{1}{M_{\star}^{2}} \dot{R}\left(n_{B}-n_{\bar{B}}\right),
$$

where $n_{B}$ and $n_{\bar{B}}$ are the baryon and antibaryon number densities respectively. Therefore the energy of a baryon is shifted by an amount of $\frac{2 \varepsilon \dot{R}}{M_{\star}^{2}}$ relative to an antibaryon. Thereby the interaction (11) dynamically violates CPT. We can assign a chemical potential to baryons: $\mu_{B}=-\mu_{\bar{B}}=-\varepsilon \frac{\dot{R}}{M_{\star}^{2}}$. So, in thermal equilibrium there will be a nonzero baryon number density given by :

$$
n_{b}=n_{B}-n_{\bar{B}}=\frac{g_{b} T^{3}}{6 \pi^{2}}\left(\pi^{2} \frac{\mu_{B}}{T}+\left(\frac{\mu_{B}}{T}\right)^{3}\right),
$$

where $g_{b} \sim \mathcal{O}(1)$ is the number of internal degrees of freedom of baryons. The entropy density of the universe is given by $s=\frac{2 \pi^{2}}{45} g_{s} T^{3}$, where $g_{s} \simeq 106$ indicates the total degrees of freedom for relativistic particles contributing to the entropy of the universe [9]. In the expanding universe the baryon number violation decouples at a temperature denoted by $T_{D}$ and a net baryon 
asymmetry remains. The ratio $\frac{n_{b}}{s}$ in the limit $T \gg m_{b}\left(m_{b}\right.$ indicates the baryon mass), and $T \gg \mu_{b}$ is then:

$$
\frac{n_{b}}{s} \simeq-\left.\varepsilon \frac{15 g_{b}}{4 \pi^{2} g_{s}} \frac{\dot{R}}{M_{\star}^{2} T}\right|_{T_{D}} .
$$

Note that in different models we may have $\dot{R}<0$ as well as $\dot{R}>0$, therefore introduction of $\varepsilon$ gives us the possibility to choose the appropriate sign for $n_{b}$.

The geometry of the universe is described by the spatially flat FRW metric

$$
d s^{2}=d t^{2}-a^{2}(t)\left(d x^{2}+d y^{2}+d z^{2}\right),
$$

where $a(t)$ is the scale factor. The Hubble parameter, $H=\frac{\dot{a}}{a}$, satisfies

$$
\begin{aligned}
H^{2} & =\frac{8 \pi G}{3} \rho \\
\dot{H} & =-4 \pi G(P+\rho) .
\end{aligned}
$$

$P$ and $\rho$ are the pressure and energy density. We assume that the universe, filled with perfect fluids, satisfies the effective equation of state: $P=\omega \rho$. The equation of state parameter, $\omega$, can be expressed in terms of the Hubble parameter: $\omega=-1-\frac{2 \dot{H}}{3 H^{2}}$. The Ricci scalar curvature is given by

$$
\begin{aligned}
R & =-6 H^{2}\left(\frac{\dot{H}}{H^{2}}+2\right) \\
& =-3 H^{2}(1-3 \omega) .
\end{aligned}
$$

From Eq. (6), it follows that

$$
\dot{R}=\frac{\sqrt{3}}{M_{p}^{3}}(1+\omega)(1-3 \omega) \rho^{\frac{3}{2}}+\frac{3}{M_{P}^{2}} \rho \dot{\omega} .
$$

$M_{p} \simeq 1.22 \times 10^{19} \mathrm{Gev}$ is the Planck mass. If $\dot{\omega}=0$, Eq. (8) reduces to the result obtained in [6].

In the following we continue our study with a universe dominated by two perfect fluids with equation of states $P_{d}=\gamma_{d} \rho_{d}$ and $P_{m}=\gamma_{m} \rho_{m}$ interacting with each other, through the source term $\Gamma_{1} \rho_{d}+\Gamma_{2} \rho_{m}$ :

$$
\begin{aligned}
\dot{\rho_{d}}+3 H\left(\rho_{d}+P_{d}\right) & =\Gamma_{1} \rho_{d}+\Gamma_{2} \rho_{m} \\
\dot{\rho_{m}}+3 H\left(\rho_{m}+P_{m}\right) & =-\Gamma_{1} \rho_{d}-\Gamma_{2} \rho_{m}
\end{aligned}
$$

Although these components don't satisfy the conservation equation solely, but

$$
\dot{\rho}+3 H(1+\omega) \rho=0
$$


Note that $\rho \simeq \rho_{m}+\rho_{d}$ and $P \simeq P_{m}+P_{d}$. $\omega$ can be written in terms of the ratio of energy densities, $r=\frac{\rho_{m}}{\rho_{d}}$,

$$
\omega=\frac{\gamma_{d}+\gamma_{m} r}{1+r}
$$

From

$$
\dot{r}=\frac{\dot{\rho_{m}}}{\rho_{d}}-r \frac{\dot{\rho_{d}}}{\rho_{d}}
$$

and Eq. (9) one can determine the behavior of $r$ with respect to the comoving time

$$
\dot{r}=-(r+1)\left(\Gamma_{1}+\Gamma_{2} r\right)+3 H r\left(\gamma_{d}-\gamma_{m}\right),
$$

or by suppressing $\gamma_{d}$

$$
\dot{r}=-(r+1)\left(\Gamma_{1}+\Gamma_{2} r\right)+3 H r(1+r)\left(\omega-\gamma_{m}\right) .
$$

$\rho$ and $r$ may be related through

$$
\frac{\dot{\rho}}{\rho}+\frac{\dot{r}+(r+1)\left(\Gamma_{1}+\Gamma_{2} r\right)}{r(r+1)\left(\gamma_{d}-\gamma_{m}\right)}\left(\gamma_{d}+1+\left(\gamma_{m}+1\right) r\right)=0 .
$$

Combining Eqs. (11) and (12) results in

$$
\dot{\omega}=\frac{\dot{\gamma_{d}}+r \dot{\gamma_{m}}}{1+r}-\frac{\left(\gamma_{m}-\gamma_{d}\right)\left(\Gamma_{1}+\Gamma_{2} r\right)}{1+r}-\frac{3\left(\gamma_{m}-\gamma_{d}\right)^{2} H r}{(1+r)^{2}} .
$$

The first and second terms show the effects of time dependence of $\gamma$ 's, and interaction of fluid components on $\dot{\omega}$, respectively. Note that even for constant $\gamma$ 's and in the absence of interactions, as the third term of (16) indicates, $\omega$ varies with time. This is due to the fact that the universe is assumed to be composed of components with different equation of state parameters. Putting Eq. (16) into Eq. (8) gives

$$
\begin{aligned}
& \dot{R}=\frac{\sqrt{3}}{M_{p}^{3}}\left(\frac{\left(1-3 \gamma_{m}\right)\left(1+\gamma_{m}\right) r^{2}+2\left(1-\gamma_{d}-\gamma_{m}-3 \gamma_{d} \gamma_{m}\right) r}{(1+r)^{2}}+\right. \\
& \left.\frac{\left(\gamma_{d}+1\right)\left(-3 \gamma_{d}+1\right)}{(1+r)^{2}}\right) \rho^{\frac{3}{2}}+\frac{3}{M_{p}^{2}}\left(\frac{\dot{\gamma_{d}}+r \dot{\gamma}_{m}}{1+r}\right) \rho- \\
& \frac{3}{M_{p}^{2}} \frac{\left(\Gamma_{1}+r \Gamma_{2}\right)\left(\gamma_{m}-\gamma_{d}\right)}{1+r} \rho-\frac{3 \sqrt{3}}{M_{p}^{3}} \frac{\left(\gamma_{m}-\gamma_{d}\right)^{2} r}{(1+r)^{2}} \rho^{\frac{3}{2}} .
\end{aligned}
$$

This equation can be rewritten in terms of $\omega$

$$
\begin{aligned}
& \dot{R}=\frac{\sqrt{3}}{M_{p}^{3}}(1+\omega)(1-3 \omega) \rho^{\frac{3}{2}}+\frac{3}{M_{p}^{2}} \frac{\left(\gamma_{m}-\omega\right) \dot{\gamma}_{d}+\left(\omega-\gamma_{d}\right) \dot{\gamma}_{m}}{\gamma_{m}-\gamma_{d}} \rho- \\
& \frac{3}{M_{p}^{2}}\left(\left(\gamma_{m}-\omega\right) \Gamma_{1}+\left(\omega-\gamma_{d}\right) \Gamma_{2}\right) \rho-\frac{3 \sqrt{3}}{M_{p}^{3}}\left(\omega-\gamma_{m}\right)\left(\gamma_{d}-\omega\right) \rho^{\frac{3}{2}}
\end{aligned}
$$


If we neglect $\dot{\omega}$, as mentioned before, only the first term remains: $\dot{R}=$ $\frac{\sqrt{3}}{M_{p}^{3}}(1+\omega)(1-3 \omega) \rho^{\frac{3}{2}}$ which is zero at $\omega=\frac{1}{3}$ and at $\omega=-1$. But by taking $\dot{\omega}$ into account we may have baryon asymmetry at $\omega=\frac{1}{3}$ and $\omega=-1$. Although the asymmetry generated during inflation will be diluted away.

It is worth to study what happens when one of the fluid components corresponds to radiation (e.g. produced after the inflation epoch). To do so, we take $\lambda_{m}=\frac{1}{3}$. In this case Eq. (17) reduces to

$$
\dot{R}=\frac{\sqrt{3}}{M_{p}^{3}} \frac{\left(1-3 \gamma_{d}\right)\left(1+\gamma_{d}\right)}{(1+r)} \rho^{\frac{3}{2}}-\frac{1}{M_{p}^{2}} \frac{\left(1-3 \gamma_{d}\right)\left(\Gamma_{1}+\Gamma_{2} r\right)}{1+r} \rho+\frac{3}{M_{p}^{2}} \frac{\dot{\gamma}_{d}}{1+r} \rho .
$$

In general $\Gamma$ 's may also be time dependent [10], e.g., one can consider $\Gamma_{1}=$ $\lambda_{1} H$ and $\Gamma_{2}=\lambda_{2} H$, where $\lambda_{1}, \lambda_{2} \in \Re$ [11. Depending on the model under consideration, the third term of (19), including the time derivative of $\gamma_{d}$, may be simplified in terms of other parameters of the model. For example consider a massive scalar field of mass $m$, with a time dependent equation of state parameter interacting with radiation. The energy density, $\rho_{d}$, and the pressure, $P_{d}$, of the scalar field satisfy

$$
\begin{aligned}
\rho_{d} & =\frac{1}{2} \dot{\phi}^{2}+\frac{1}{2} m^{2} \phi^{2} \\
P_{d} & =\frac{1}{2} \dot{\phi}^{2}-\frac{1}{2} m^{2} \phi^{2} .
\end{aligned}
$$

The time dependent equation of state parameter of the scalar field is then

$$
\gamma_{d}=\frac{\frac{1}{2} \dot{\phi}^{2}-\frac{1}{2} m^{2} \phi^{2}}{\frac{1}{2} \dot{\phi}^{2}+\frac{1}{2} m^{2} \phi^{2}} .
$$

The scalar field interacts with another component (radiation) characterized by the equation of state parameter $\gamma_{m}=\frac{1}{3}$ :

$$
\begin{aligned}
\dot{\rho}_{d}+3 H\left(\rho_{d}+P_{d}\right) & =\Gamma_{1} \rho_{d}+\Gamma_{2} \rho_{\mathcal{R}} \\
\dot{\rho}_{\mathcal{R}}+4 H \rho_{\mathcal{R}} & =-\Gamma_{1} \rho_{d}-\Gamma_{2} \rho_{\mathcal{R}}
\end{aligned}
$$

The subscript $\mathcal{R}$ denotes the component with $\gamma_{m}=\frac{1}{3}$. To determine $\dot{\gamma}_{d}$ in Eq. (19), let us define $u=\left(1-\gamma_{d}\right) \frac{\rho_{d}}{2}$. Then using

$$
\dot{u}=m \rho_{d} \sqrt{1-\gamma_{d}^{2}}
$$

which was verified in [12], we are led to

$$
\dot{\gamma}_{d}=-2 m \sqrt{1-\gamma_{d}^{2}}+\left(1-\gamma_{d}\right)\left(\Gamma_{1}+r \Gamma_{2}\right)-3 H\left(1-\gamma_{d}^{2}\right) .
$$

By supposing

$$
\Gamma_{1}=3 \lambda_{d} H, \quad \Gamma_{2}=3 \lambda_{\mathcal{R}} H, \quad \lambda_{d}, \lambda_{\mathcal{R}} \in \Re,
$$


we arrive at

$$
\dot{\gamma}_{d}=-2 m \sqrt{1-\gamma_{d}^{2}}+3 H\left(1-\gamma_{d}\right)\left(\lambda_{d}+\lambda_{\mathcal{R}} r-\gamma_{d}-1\right) .
$$

Finally we deduce

$$
\dot{R}=-\frac{6 m}{M_{p}^{2}} \frac{\sqrt{1-\gamma_{d}^{2}}}{1+r} \rho+\frac{2 \sqrt{3}}{M_{p}^{3}} \frac{\left(-1-\gamma_{d}+\lambda_{d}+r \lambda_{\mathcal{R}}\right)}{1+r} \rho^{\frac{3}{2}} .
$$

If the potential is negligible with respect to the kinetic energy, $\dot{\phi}^{2} \gg m^{2} \phi^{2}$, we have $\gamma_{d} \simeq 1$ and $\dot{\gamma}_{d} \simeq 0$. In this case $\dot{R}=\frac{2 \sqrt{3}}{M_{p}^{3}}\left(\frac{-2+\lambda_{d}+r \lambda_{\mathcal{R}}}{1+r}\right) \rho_{d}^{\frac{3}{2}}$.

When the scalar field dominates, i.e., in the limit $r \rightarrow 0, \dot{R}=-\frac{6 m}{M_{p}^{2}} \sqrt{1-\gamma_{d}^{2}} \rho_{d}+$ $\frac{2 \sqrt{3}}{M_{p}^{3}}\left(-1-\gamma_{d}+\lambda_{d}\right) \rho_{d}^{\frac{3}{2}}$.

In the limit $r \rightarrow \infty$, i.e, when $\rho_{\mathcal{R}}$ dominates

$$
\dot{R}=\frac{2 \sqrt{3}}{M_{p}^{3}} \lambda_{\mathcal{R}} \rho_{\mathcal{R}}^{\frac{3}{2}} .
$$

So, even in the radiation dominated era, we can have $\dot{R} \neq 0$ if $\lambda_{\mathcal{R}} \neq 0$. For radiation component, the energy density is related to the equilibrium temperature via 9 ]

$$
\rho_{\mathcal{R}}=\epsilon_{\mathcal{R}} T^{4},
$$

where $\epsilon_{\mathcal{R}}=\frac{\pi^{2}}{30} g_{\star}$ and $g_{\star}$ counts the total number of effectively massless degrees of freedom. Its magnitude is $g_{\star} \simeq g_{s}$. Therefore the baryon asymmetry in terms of temperature can be determined from (4), (28), and (29):

$$
\frac{n_{b}}{s} \simeq 2.5 g_{b} \lambda_{\mathcal{R}} \frac{T_{D}^{5}}{M_{\star}^{2} M_{p}^{3}} .
$$

By defining $M_{\star}=\alpha M_{p}$ we find that $\frac{n_{b}}{s}$ is of order

$$
\frac{n_{b}}{s} \sim \frac{\lambda_{\mathcal{R}}}{\alpha^{2}}\left(\frac{T_{D}}{M_{p}}\right)^{5}
$$

When $\gamma$ 's are time dependent, in general, derivation of the exact solutions of Eq. (22) is not possible. Thus explaining the precise behavior of the fluid components in terms of temperature, except in special situations like (30), is not straightforward. But when $\gamma$ 's are constant, it may be possible to determine baryogenesis in terms of decoupling temperature. We will study this situation through two examples.

As a first example consider $\gamma_{m}=\gamma_{\mathcal{R}}=1 / 3$. We assume that $\gamma_{d}$ is a constant satisfying $\gamma_{d}>\frac{1}{3}$ corresponding to a non-thermal component 
decreasing more rapidly than radiation [6]. If there is no energy exchange, i.e, $\Gamma_{1}=\Gamma_{2}=0$ :

$$
\begin{aligned}
\dot{\rho_{d}}+3\left(\gamma_{d}+1\right) H \rho_{d} & =0 \\
\dot{\rho}_{\mathcal{R}}+4 H \rho_{\mathcal{R}} & =0 .
\end{aligned}
$$

In this case

$$
\dot{R}=\frac{\sqrt{3}}{M_{p}^{3}} \frac{\left(1-3 \gamma_{d}\right)\left(1+\gamma_{d}\right)}{(1+r)} \rho^{\frac{3}{2}} .
$$

This result may reproduced by taking $\gamma_{d}=1$ (which results in $\dot{\gamma}_{d}=0$ ) and $\lambda_{\mathcal{R}}=\lambda_{d}=0$ in (27). $r$ satisfies

$$
\dot{r}=\operatorname{Hr}\left(3 \gamma_{d}-1\right),
$$

hence, $r$ is an increasing function of comoving time, i.e., $\rho_{d}$ decreases faster than the radiation component. $\dot{R}$ is also an increasing function of time. To see this let us consider

$$
\ddot{R}=\frac{1}{2 M_{p}^{4}}\left(1+\gamma_{d}\right)\left(3 \gamma_{d}-1\right)\left(9\left(\gamma_{d}+1\right)+2 r\left(3 \gamma_{d}+5\right)\right) \frac{\rho^{2}}{(1+r)^{2}} .
$$

which was obtained using Eqs. (15) and (33). Hence $\gamma_{d}>\frac{1}{3}$ leads to $\ddot{R}>0$.

Eq. (32) implies $\rho_{\mathcal{R}} \propto a^{-4}$, hence the temperature redshifts as $T \propto a^{-1}$, this relation and Eq. (32) imply $\rho_{d} \propto T^{3\left(1+\gamma_{d}\right)}$. If at a temperature denoted by $T=T_{R D}, \rho_{\mathcal{R}}=\rho_{d}$, we can write

$$
\rho_{d}=\epsilon_{\mathcal{R}} T_{R D}^{4}\left(\frac{T}{T_{R D}}\right)^{3\left(1+\gamma_{d}\right)},
$$

which results in

$$
\rho=\epsilon_{\mathcal{R}} T_{R D}^{4}\left(\left(\frac{T}{T_{R D}}\right)^{4}+\left(\frac{T}{T_{R D}}\right)^{3\left(1+\gamma_{d}\right)}\right),
$$

and

$$
r=\left(\frac{T}{T_{R D}}\right)^{1-3 \gamma_{d}} .
$$

by putting Eqs. (37) and (38), in Eq. (33) and subsequently in Eq. (4) at $T=T_{D}$, the baryon asymmetry may be determined:

$$
\frac{n_{b}}{s} \simeq \frac{1.28 g_{b}}{M_{\star}^{2} M_{p}^{3}}\left(1+\gamma_{d}\right)\left(3 \gamma_{d}-1\right) \frac{T_{R D}^{6}}{T_{D}}\left(\frac{T_{D}}{T_{R D}}\right)^{\frac{9\left(1+\gamma_{d}\right)}{2}} \sqrt{1+\left(\frac{T_{D}}{T_{R D}}\right)^{1-3 \gamma_{d}}} .
$$

In the limit $r \rightarrow 0$ (related to $\left.T_{D} \gg T_{R D}\right)$,

$$
\frac{n_{b}}{s} \simeq \frac{1.28 g_{b}}{M_{\star}^{2} M_{p}^{3}}\left(3 \gamma_{d}-1\right)\left(1+\gamma_{d}\right) \frac{T_{R D}^{6}}{T_{D}}\left(\frac{T_{D}}{T_{R D}}\right)^{\frac{9\left(1+\gamma_{d}\right)}{2}},
$$


which is the same as the result obtained in [6], where the effect of time dependence of $\omega$ was ignored, indeed in this limit $\dot{\omega}$ does not contribute in baryon asymmetry. In the limit $r \gg 1$ which corresponds to $\rho_{d} \ll \rho_{\mathcal{R}}$ we have

$$
\frac{n_{b}}{s} \simeq \frac{1.28 g_{b}}{M_{\star}^{2} M_{p}^{3}}\left(1+\gamma_{d}\right)\left(3 \gamma_{d}-1\right) \frac{T_{R D}^{6}}{T_{D}}\left(\frac{T_{D}}{T_{R D}}\right)^{5+3 \gamma_{d}} .
$$

E. g. if we define $T_{D}=\beta T_{R D}, \beta \ll 1$, and as before $M_{\star}=\alpha M_{p}$, then the order of magnitude of $\frac{n_{b}}{s}$ is obtained as

$$
\frac{n_{b}}{s} \sim\left(3 \gamma_{d}-1\right) \frac{\beta^{4+3 \gamma_{d}}}{\alpha^{2}}\left(\frac{T_{R D}}{M_{p}}\right)^{5} .
$$

As a second example consider a model, with constant $\gamma_{d}$, and $\gamma_{m}=\frac{1}{3}$. Also assume that at least one of the constant $\Gamma$ 's are not zero. Hence

$$
\dot{R}=\frac{\sqrt{3}}{M_{p}^{3}} \frac{\left(1-3 \gamma_{d}\right)\left(1+\gamma_{d}\right)}{(1+r)} \rho^{\frac{3}{2}}-\frac{1}{M_{p}^{2}} \frac{\left(1-3 \gamma_{d}\right)\left(\Gamma_{1}+\Gamma_{2} r\right)}{1+r} \rho,
$$

may be used to determine $\frac{n_{b}}{s}$. In this situation although Eq. (29) holds but Eq. (36) is no longer valid. The temperature does not behave as $T \propto a^{-1}$ and, instead, we have $T=f(a)$ [9], [13]. In some special cases, $\dot{R}$ can be easily determined in terms of decoupling temperature. For example, at $r \simeq 1$ (when $\omega \simeq \frac{3 \gamma_{d}+1}{6}$ ) we have

$$
\rho \simeq 2 \epsilon_{\mathcal{R}} T^{4}
$$

and

$$
\frac{n_{b}}{s} \simeq \frac{\varepsilon g_{b}}{M_{\star}^{2}}\left(\frac{3.6}{M_{p}^{3}}\left(3 \gamma_{d}-1\right)\left(1+\gamma_{d}\right) T_{D}^{5}-\frac{0.25}{M_{p}^{2}}\left(3 \gamma_{d}-1\right)\left(\Gamma_{1}+\Gamma_{2}\right) T_{D}^{3}\right) .
$$

Note that in the above example $T_{D}=T_{R D}$.

Also For $r \rightarrow 0$ and $r \rightarrow \infty$, corresponding to $\omega \simeq \gamma_{d}$ and $\omega \simeq \gamma_{m}$ respectively, Eq. (16) leads to

$$
\dot{\omega}= \begin{cases}\left(\gamma_{d}-\gamma_{m}\right) \Gamma_{1}, & r \rightarrow 0 \\ \left(\gamma_{d}-\gamma_{m}\right) \Gamma_{2}, & r \rightarrow \infty\end{cases}
$$

Therefore for $\gamma_{m}=\frac{1}{3}$,

$$
\dot{R}=\left\{\begin{array}{l}
\frac{\sqrt{3}}{M_{p}^{3}}\left(1+\gamma_{d}\right)\left(1-3 \gamma_{d}\right) \rho_{d}^{\frac{3}{2}}-\frac{\left(1-3 \gamma_{d}\right) \Gamma_{1}}{M_{p}^{2}} \rho_{d}, \quad r \rightarrow 0 \\
\frac{\left(3 \gamma_{d}-1\right) \Gamma_{2}}{M_{p}^{2}} \rho_{\mathcal{R}}, \quad r \rightarrow \infty .
\end{array}\right.
$$

E.g., if one suppose that the reheating process after the inflation is due to the decay of a scalar field (the inflaton field: $\phi$ ) to (ultra)relativistic species, via coherent oscillation [9], then

$$
\begin{aligned}
\dot{\rho}_{\phi}+3 H \rho_{\phi}^{2} & =-\Gamma_{\phi} \rho_{\phi} \\
\dot{\rho}_{R}+4 H \rho_{\mathcal{R}} & =\Gamma_{\phi} \rho_{\phi},
\end{aligned}
$$


where $\rho_{\mathcal{R}}$ is the energy density in the relativistic decay products. If $M^{4}$ is the vacuum energy of the scalar field at the beginning of the oscillation, from $t \simeq \frac{M_{p}}{M^{2}}$ until $t \simeq \Gamma_{\phi}^{-1}, \phi$ particles, which behave like non-relativistic particles (i.e., $\gamma_{\phi}=0$ ), dominate the energy density [9]. During this time, $a(t) \propto t^{\frac{2}{3}}, \rho \propto a^{-3}$ and

$$
\dot{R}=\frac{\sqrt{3}}{M_{p}^{3}} \rho_{\phi}^{\frac{3}{2}}+\frac{\Gamma_{\phi}}{M_{p}^{2}} \rho_{\phi}
$$

Although $\rho_{\mathcal{R}} \propto T^{4}$ and Eq. (29) is still valid, but due to the interaction term, $\rho_{\mathcal{R}} \propto a^{-4}$ does not hold. Then $T \propto a^{-1}$ is not valid. In this case one can show that the temperature can be approximated by 13 .

$$
T=T_{R D}\left(\frac{a_{R D}}{a}\right)^{\frac{3}{8}} .
$$

Hence at the time of decoupling

$$
\rho_{\phi}=\epsilon_{\mathcal{R}} T_{R D}^{4}\left(\frac{T_{D}}{T_{R D}}\right)^{8},
$$

and

$$
\frac{n_{b}}{s} \simeq \frac{\varepsilon}{M_{\star}^{2}} \frac{g_{b}}{8}\left(\frac{\sqrt{3 \epsilon_{\mathcal{R}}}}{M_{p}^{3}} T_{R D}^{-1} T_{D}^{6}+\frac{\Gamma_{\phi}}{M_{p}^{2}} T_{R D} T_{D}^{2}\right) .
$$

To derive the above equation we have multiplied our result by the dilution factor $\left(\frac{T_{R D}}{T_{D}}\right)^{5}$ [6]. The first term is the same as the result obtained in [6], where $\dot{\omega}$ was ignored. If we take $\Gamma_{\phi} \sim \frac{T_{R D}^{2}}{M_{p}}$, and as before define $M_{\star}=\alpha M_{p}$ and $T_{D}=\beta T_{R D}$, then we are able to compare the order of magnitudes of terms contributing in (52):

$$
\begin{aligned}
\frac{1}{M_{\star}^{2}} \frac{g_{b}}{8}\left(\frac{\sqrt{3 \epsilon_{\mathcal{R}}}}{M_{p}^{3}} T_{R D}^{-1} T_{D}^{6}\right) & \sim 10 \frac{\beta^{6}}{\alpha^{2}}\left(\frac{T_{R D}}{M_{p}}\right)^{5}, \\
\frac{1}{M_{\star}^{2}} \frac{g_{b}}{8}\left(\frac{\Gamma_{\phi}}{M_{p}^{2}} T_{R D} T_{D}^{2}\right) & \sim \frac{\beta^{2}}{\alpha^{2}}\left(\frac{T_{R D}}{M_{p}}\right)^{5} .
\end{aligned}
$$

When $\beta \gg 1$ the second term in (52) which comprises the effect of decay width is negligible.

At the end let us note that if $\omega$ were time independent, $\frac{n_{b}}{s}$ would be vanish in radiation dominated era. But by taking $\dot{\omega}$ into account, it is easy to see that if $\Gamma_{2} \neq 0$, in the limit $\rho_{\mathcal{R}} \gg \rho_{\phi}$ we have $\dot{R}=\frac{\left(3 \gamma_{d}-1\right) \Gamma_{2}}{M_{p}^{2}} \rho_{\mathcal{R}}$, therefore

$$
\frac{n_{b}}{s} \simeq-\varepsilon \frac{g_{b}}{8 M_{\star}^{2}} \frac{\left(3 \gamma_{d}-1\right) \Gamma_{2}}{M_{p}^{2}} T_{D}^{3} .
$$

Thereby $\frac{n_{b}}{s} \sim \frac{\left(3 \gamma_{d}-1\right)}{\alpha^{2}} \frac{\Gamma_{2} T_{D}^{3}}{M_{p}^{4}}$. 


\section{References}

[1] C. Bennett et al., Astrophys. J. Suppl. 148, 1 (2003).

[2] A. G. Cohen, A. D. Rujula and S. L. Glashow, Astrophys. J. 495, 539 (1998).

[3] S. Burles, K. M. Nollet and M. S. Turner, Phys. Rev. D 63, 063512 (2001).

[4] A. D. Sakharov, JETP Letters 5, 24 (1967).

[5] A. G. Cohen and D. B. Kaplan, Phys. Lett. B 199, 251 (1987).

[6] H. Davoudiasl, R. Kitano, G. D. Kribs, H. Murayama and P. J. Steinhardt, Phys. Rev. Lett. 93, 201301 (2004).

[7] G. L. Alberghi, R. Casadio and A. Tronconi, Mod. Phys. Lett. A 22, 339 (2007)(arXiv:hep-ph/0310052 v4); H. Li, M. Li and X. Zhang, Phys. Rev. D 70, 047302 (2004); T. Shiromizu and K. Koyama, J. Cosmol. Astropart. Phys. 0407 (2004) 011; B. Feng, H. Li, M. Li and X. Zhang, Phys. Lett. B 620, 27 (2005); M. C. Bento, R. G. Felipe and N. M. C. Santos, Phys. Rev. D 71, 123517 (2005); S. va der Post and T. Prokopec, hep-ph/0612313 G. Lambiase, Phys. Lett. B 642, 9 (2006); G. Lambiase and S. Mohanty, astro-ph/0611905.

[8] G. Lambiase and G. Scarpetta, Phys. Rev. D 74, 087504 (2006).

[9] E. W. Kolb and M. S. Turner, The Early Universe, Westview Press, (1994).

[10] E. W. Kolb, A. Notari and A. Riotto, Phys. Rev. D 68, 123505 (2003).

[11] W. Zimdahl and D. Pavon, Phys. Lett. B 521, 133 (2001); L. P. Chimento, A. S. Jakubi, D. Pavon and W. Zimdahl, Phys. Rev. D 67, 083513 (2003); E. Majerotto, D. Sapone, and L. Amendola, astro-ph/0410543 Z. K. Guo, R. G. Cai and Y. Z. Zhang, J. Cosmol. Astropart. Phys. 05 (2005) 002; R. G. Cai and A. Wang, J. Cosmol. Astropart. Phys. 03 (2005) 002; John D. Barrow and T. Clifton, Phys. Rev. D 73, (2006) 103520; H. Mohseni Sadjadi and M. Honardoost, Phys. Lett. B 647, 231 (2007); H. Mohseni Sadjadi, J. Cosmol. Astropart. Phys. 02 (2007) 026.

[12] H. Mohseni Sadjadi and M. Alimohammadi, Phys. Rev. D 74, 103007 (2006).

[13] S. Davidson, M. Losada and A. Riotto, Phys. Rev. Lett. 84, 4284 (2000). 